\title{
HIV/AIDS Among Injecting Drug Users: A review on Epidemiology and Management of Occupational Exposure in Iranian Health Network Setting
}

\author{
Seyed Mohammad Alavi ${ }^{1, *}$, Mohammad Hossein Sarmast Shushtari ${ }^{2}$ \\ ${ }_{1}^{1}$ Infectious and Tropical Diseases Research Center, Jundishapur University of Medical Sciences, Ahvaz, IR Iran \\ ${ }^{2}$ Medical School, Jundishapur University of Medical Sciences, Ahvaz, IR Iran \\ ${ }^{*}$ Corresponding author: Seyed Mohammad Alavi, Infectious and Tropical Diseases Research Center, Jundishapur University of Medical Sciences, Ahvaz, IR Iran. Tel:+986113387724, Fax: \\ 06113335396, E-mail: alavi.seyedmohammad@yahoo.com
}

Received: June 25, 2012; Revised: October 30, 2012; Accepted: December 15, 2012

\begin{abstract}
Context: Human Immunodeficiency Virus (HIV) infection and acquired immunodeficiency syndrome (AIDS) concentrated in injecting drug users (IDUs) is a major public health in Iran as well as throughout the world. Health care workers(HCW) are occupationally at the risk of HIV infection. The aim of this article is to review the information about the IDUs, epidemiology, diagnosis, natural course of infection, immunopathogenesis, and occupational risks associated with managing HIV in the health care workplace.

Evidence Acquisition: Information obtained from previous investigation on HIV infection has yielded a better knowledge about HIV. Results: Because HCWs are at the risk of HIV infection from IDUs attending the health care units, knowledge about preventive strategy and the efficacy of post exposure anti-viral therapy enables general physicians to manage these every moment events.

Conclusions: Based on existing data, HIV infection surveillance, performance of standard precaution, and post exposure prophylaxis with anti-retroviral drugs outlined in this review article represent reasonable interim approaches to this complex problem.
\end{abstract}

Keywords: HIV; Drug Abuse; Occupational exposure; Health Personnel

\section{Context}

A high percentage of HIV infected population throughout the world are injecting drug users (IDUs), the majority of them are living in undeveloped countries. More than two third of HIV/AIDS persons in Iran are attributed to IDUs (1). In addition to HIV infection these people are also infected with serious viruses including viral hepatic pathogens such as hepatitis B virus (HBV) and hepatitis $\mathrm{C}$ virus (HCV) due to sharing contaminated needles and syringes (2-6). Beside the mentioned health problems, IDUs are exposed to dangerous infections such as tuberculosis (TB) and multi-drug TB (MDR-TB) (7-12), sexually transmitted infection (STI) (13), and malaria (14) because of their antisocial behavior and imprisonment $(8,14-16)$. One particular cause of STI transmission among female sexworkers is unprotected sexual relationships with IDUs (17), who serve as reservoirs for HIV, HCV, or HBV (18), in addition to their high-risk sexual (17) and injecting (19) behaviors. The considerable prevalence of HIV and HCV with the rate of approximately $14 \%$ and $80 \%$, respectively among Iranian IDUs at the end of 2009 (20) suggests the important role for this population in transmission of these infections to the general population. Therefore, IDUs are the most important source of dangerous infec- tious pathogens threating the public health (21). General physicians as well as other health care workers (HCWs) concerned with public health should not overlook the dangers of IDUs in health care settings. The aim of this article is to review the current information about epidemiology, diagnosis, and prevention of HIV/AIDS among IDU person attending health care workplace.

\section{Evidence Acquisition}

A systematic review of the literature on the epidemiology, diagnosis, and prevention of HIV from 1991 to 2012 using computerized bibliographic databases which include Pub Med, Current Content, Scopus, EMBASE, Google Scholar, and Iran Medex was carried out to increase understanding of HIV/AIDS in health care settings.

\section{Results}

\subsection{Illicit Drug Use}

Although addiction to opium has been present in Iran for centuries, now, because of emergence of intravenous drug use along with transmission of infectious diseases including HIV and HCV it has changed into a major pub-

Implication for health policy/practice/research/medical education: This article is a useful guide for general physician involved in public health to manage infectious diseases.

Copyright @ 2013, Ahvaz JundishapurUniversity of Medical Sciences; Published by Kowsar Corp. This is an open-access article distributed under the terms of the Creative Commons Attribution License, which permits unrestricted use, distribution, and reproduction in any medium, provided the original work is properly cited. 
lic health with significant social, psychological, familial, and economic importance $(22,23)$.

\subsection{Profile of Drug Addicts in Iran}

Data from various provinces of Iran and within different social groups show that more than $90 \%$ of the drugs abusing population are male. Around two-thirds of the addict population are married, and about less than 10\% of the addicts live alone. The majority of drug abusers are employed, where the unemployed comprise only a fifth of this population $(22,23)$.

\subsection{Kind of Substances}

Narcotics are the most common substance in Iran with the incidence rate of $73 \%$ (some of them also abuse a preparation of opium called Shireh), heroin ranked second with $39 \%$, cannabis use with the rate of $13 \%$, and cocaine and stimulant with the negligible rate. On average, $62 \%$ of them are uni-drug users (22).

\subsection{Route of Substance Administration}

Opium and Shireh are abused via smoking, ingestion, or rarely by injection, while heroin is sniffed, smoked, or injected. Approximately 20 - $25 \%$ of total Iranian addicts abuse their substances via intravenous injection $(1,2,22)$. Despite easy access to needles in Iran, high proportion of IDUs mostly in street and in prisons had a positive history of sharing syringes and needles with their friends. It appears that the sharing is related to a practice of injecting with a peer group. Two-thirds of IDUs claimed to have engaged in this practice, therefore it is estimated that about $66.6 \%$ of them share needles (23).

\subsection{Health Care Network in Iran}

Ministry of Health and Medical Education of Iran, through its network of universities of medical sciences including health centers in the country, is in charge of provision of healthcare services. An elaborate system of health network has been established which has ensured provision of primary health services to the vast majority of public $(24,25)$.

\subsubsection{Primary Health Center}

The Primary Health Center (PHC) is the fundamental unit of the public health system providing services throughout Iran, from remote mountain areas to inner urban areas in the country's capital (24). The Iranian Health Care Network provides health services through first line health services called in Iran" Khaneh-e-Behdasht", health centers, and tertiary hospitals $(24,25)$. Since 1984, the activities of the health system have resulted in a dramatic decrease in the burden of common and endemic infectious diseases (25).

\subsection{Epidemiology of HIV/AIDS}

HIV/AIDS is amongst the most serious health problems worldwide and has developed important challenges. The first cases were reported in 1981, and today HIV not only affects the health of populations, it impacts households, communities, sociocultural development, and economic growth of nations (20).

\subsubsection{Global HIV/AIDS Status}

Now, with the progress made in the HIV/AIDS control in the world, the trend of HIV/AIDS has decreased. Today, there are approximately 34 million people living with HIV and nearly 30 million people have died of AIDS-related causes since 1981. Although a lot of progress have been made in the recent years by HIV testing and awareness of HIV status leading to a better personal control on the infection, but still many people are unaware of their HIV status $(20,26)$.

\subsubsection{HIV/AIDS Status in Asia}

Due to the growing trend of Iranians traveling to Asian countries such as India, China, Malaysia, and Thailand, and the geographical Iranian neighborhood with Pakistan, Afghanistan, and Central Asian countries, it is important to know the HIV status in those regions (20). Approximately 5 million HIV infected people are living in South/South-East Asia and East Asia. While most national epidemics are supposed to be stabilized, HIV prevalence is increasing in Bangladesh, Pakistan, and the Philippines. The most populous nations in the world (China and India) with large numbers of HIV infected people are located in the region near to Iran (20).

\subsubsection{Epidemiology of HIV Infection in the Middle East and North Africa}

There is considerable evidence on HIV prevalence and risk behavior practices among IDUs, men having sex with men (MSM), and female sex workers (FSWs) in this region. IDU is a key HIV mode of transmission worldwide (27) and was found to be the dominant mode of transmission in several countries such as Iran (28). Overall, HIV prevalence among IDUs was in the low to intermediate range compared to global figures (29), with significant variation across and within these countries. Although the prevalence of HIV among this risk group was low in several countries, there was robust evidence for concentrated epidemics among IDUs in at least Iran and Pakistan (30). Concentrated epidemics are defined as HIV prevalence consistently exceeds $5 \%$ in a high-risk group, while remaining below $1 \%$ in pregnant women (31). HIV incidence was found to be at very high levels of $16.8 \%$ per person every year among IDUs in a detention center in Iran (32). MSM are the most hidden and stigmatized of all HIV-risk groups in the region. Although reliable estimates of the 
Alavi SM et al.

number of MSM are scarce, available data indicate that the proportion of the male population engaging in anal sex with males in this region is comparable to global levels of roughly $2-3 \%(33,34)$. The prevalence of HIV among FSWs in the region continues to be at low levels in most countries, although at levels much higher than those in the general population.

\subsubsection{Epidemiology of HIV Infection in Iran}

According to statistics collected from medical and health services to the date of June 2012, total of 24290 persons with HIV/AIDS in the country have been identified, where $90.8 \%$ of them were men and 9.2\% were women. 3455 persons have been identified to have AIDS and 4722 patients with AIDS have died, and $46.4 \%$ of patients with HIV infection are in the age group 25 - 34 years. The common way of transmission was $69.6 \%$ by IDU, $10.5 \%$ by sex, blood transfusion and blood products (1\%), and from mother to child transmission (1\%). Transmission in $17.9 \%$ of this group was unknown (35).

\subsection{Route of Transmission}

The most common way of transmission of HIV is through heterosexual contact. Other ways, such as homosexual contact in men, female sex working, sharing injection equipments among injecting drug users, and mother to child are also important (26). Persons at highest risk for development of HIV/AIDS related diseases are FSWs (36), MSM (34), IDUs, IDUs sex partner (27, 30), and HCWs who are at risk for needle-stick and other exposures (37). The risk of HIV transmission by a deep hollow needle injury from an HIV infected source is about $0.3 \%$, but if the source is HIV negative with a high risk behavior then the risk is lower (38). Studies show that the risk of HIV transmission is lower than that of HCV and HBV with the rate of $3 \%$ and $30 \%$, respectively $(26,38)$. Mother-to-child HIV transmission rate is $30-40 \%$, but when the mother is HCV co-infected this rate is higher. This risk increases with higher maternal viral load. Breast feeding transmits HIV to infant (39). HIV transmission is under influence of mode of delivery, so HIV infection is a contraindication for pregnancy, therefore HIV testing should be considered as a routine screening test in pregnant women (26).

\subsubsection{Transmission of HIV in the Health Care Units}

\subsubsection{Infection Acquired Through the Medical Interven- tions}

Medically transmitted infection is said to infections that are caused by medical interventions such as blood/ blood products transfusion or organ transplantation. Blood borne pathogens such as HIV can be transmitted by transfusion of contaminated blood or blood products and by seropositive organ transplant donors (26).

\subsubsection{Occupational Exposure}

\subsection{Patient-to-HCW Transmission}

HIV is a blood-borne viral infection that can be transmitted by transfusion or needle stick. By this reason, the HCWs are occupationally at the risk of HIV transmission from infected patients. Percutaneous exposure to blood is the primary mode of transmission for HIV from patients to health staff(26). Splashes of blood from infected patients to HCWs' mucous membranes are reported to be a route of transmission for HIV (40).

\subsection{Patient-to-patient Transmission}

Transmission of HIV in health care setting deserves special emphasis. Nosocomial transmission of HIV among patients and staff is described in the past reports from various countries. Hemodialysis settings, cardiovascular departments, endoscopic clinics, and orthopedic wards are hospital divisions where HIV transmission has been reported $(26,27)$. Spreading in these units has been suggested to be due to environmental contamination, contaminated dialysis machines, and inadequate infection control procedures in the dialysis unit (26). In addition, patient-to-patient transmission in health care settings, primarily related to faulty injection practices, appears to be a reasonably important mode of HIV transmission in developing countries (41).

\subsection{HCW-to-Patient Transmission}

To date, iatrogenic transmission of HIV from HIV-infected HCW to their patients in some countries has been reported. The first report of documented iatrogenic transmission in dental surgery was reported from USA (42).

\subsection{Immunopathogenesis of HIV Infection}

The main effect of HIV infection is progressive reduction in the number of T lymphocytes CD4+ which is resulted in progressive decline in the immunity system (43).

\subsection{The Natural Course of HIV Infection}

1. Acute infection or acute retroviral syndrome: This occurs 2 - 4 weeks after exposure to the virus. At this stage, patients experience symptoms such as fever, rash, joint pain, and enlarged lymph nodes. At this stage, which is self-limited, serological tests are negative and the diagnosis of acute infection is based on detection of viral RNA through the plasma $(27,43)$.

2. Asymptomatic acute infection: Since the start of the infection to onset of AIDS, about 10 years in adults and 2 years in children, the patients has no symptoms and appear healthy (43).

3. Persistent generalized lymphadenopathy (PGL): PGL is called to large lymph nodes in more than 2 site of body 
except the groin area. Enlarged lymph nodes are nonsymmetrical and non-painful (43).

4. Immunosuppression period: Along with the progress of HIV infection, patients are prone to serious infections such as tuberculosis, pneumonia, and repeated fungal and viral infections. Some patients present general symptoms such as fever and weight loss, which was called in the past as ARC. The others present diarrhea and weight loss, which was called Slime disease. Finally, patients lost their lives due to certain infections such as cryptococcal meningitis or kaposi sarcoma, in which stage infection is categorized as ull blown disease $(10,26,44)$.

\subsection{HIV Testing}

Examination of HIV antibodies in the blood is used for routine HIV testing. It is recommended that two tests be performed to detect HIV infection. For diagnosis of infection, both HIV antibody tests should be positive. Western blot (WB) test should be performed to confirm the infection, but in limited resource area (lacking access to WB testing facilities) two positive Elisa-Anti-HIV tests for riskassessed patients is also accepted for diagnosis of HIV infection $(10,39,45)$. Due to the limitation of facilities and resources in health work places, other new antigen detection based tests are not discussed here.

\subsection{Case Definition}

According to WHO case definition for AIDS surveillance, in limited resource areas (lacking access to HIV testing facilities) AIDS patients are defined as bellow:

\subsubsection{Adults}

In adults case definition of AIDS is definite when at least two major and one minor symptom are present (44). Major symptoms include: 1) Weight loss of over $10 \%$ of body weight. 2) Diarrhea lasting more than 1 month. 3) Fever lasting more than 1 month. Minor symptoms include: 1) A cough that lasts more than 1 month (excluding tuberculosis).2) Diffuse pruritic dermatitis. 3) A history of herpes zoster. 4) Oro-pharyngeal candidiasis. 5) Disseminated chronic herpes simplex. 6) Diffuse lymphadenopathy.

\subsubsection{Children}

In children case definition of AIDS is definite when at least two major and two minor symptoms are present (44):Major symptoms include:1) Weight loss or abnormal growth. 2) Diarrhea lasting more than 1 month. 3) Fever lasting more than 1 month. Minor symptoms include:1) A lasting cough. 2) Diffuse rash. 3) Repeated ear or pharyngeal infections. 4) Oro-pharyngeal candidiasis. 5) Diffuse lymphadenopathy.

Prevention of HIV infection in health care units:

\subsection{Standard Precautions}

In dealing with every patient as well as HIV infected patients, following precautions should be taken $(26,46)$ :

1. Use gloves while contacting the patient and wash hands after that.

2. Use protective eyewear and mask.

3. Use sharp materials safely and cautiously.

4. Clean the blood stains and contaminated materials on surfaces with appropriate disinfectants.

5. Use standard disinfection and sterilization.

6. Treat any material soiled with blood or body substance as contaminated.

7. Vaccinate all the clinical and laboratory staff against hepatitis $\mathrm{B}$.

8. Other measures include: wearing two pairs of gloves over each other, changing surgical methods to prevent exposure prone to infection, use safe systems without needles and other sharp tools.

\subsection{HIVTreatment}

HIV treatment includes the use of combination of antiretroviral therapy against the virus itself and medications to prevent and treat the many opportunistic infections that can occur when the immune system is compromised by HIV. Combination anti retroviral treatment (ART), first introduced in 1996, has led to dramatic reductions in morbidity and mortality, and access has increased in recent years, rising from less than half a million people on treatment in 2001 to 6.6 million people in 2010, with an increase of 1.35 million people since 2009 (26).

\subsection{Preventive Measures}

\subsubsection{Pre-Exposure}

Standard precaution, to avoid contact with potentially contaminated body substances such as blood, bloody secretions, or tissues of patients in health care units, should be performed.

\subsubsection{Post Exposure}

Approach to occupational exposure to potentially contaminated body substances should be avoided. In the case of occupational exposure including splashes, mucus membrane, or needle sticks preventive recommendations should be followed as below $(26,46)$ :

1. Appropriately wash the exposed area.

2. Inform the event to in charge health official.

3. Test the source patient for HIV and hepatitis B and C.

4. If the source patient was infected, the contact person should be tested for above mentioned tests.

5. If the exposed person was unvaccinated to hepatitis $B$ vaccine, hepatitis B immunoglobulin has to be injected and the primary HBV vaccination has to be given with a preferable booster dose.

6. If skin or intravenous contact with HIV is confirmed, 
in the first few hours prophylaxis with anti-retroviral drug must be given and consultation with an infectious disease specialist should be done. Combination therapy with at least 3 anti-retroviral drugs for 4 weeks (according to national HIV program) is recommended (35).

7. Protect sexual partners through a 12-week follow-up period and prevent from pregnancy and blood donation and report every febrile illness.

8. Repeat anti-HIV tests in weeks 6 to 12.

9. Be sure of reporting the events, results of the tests, and the follow-up.

\section{Conclusion}

Based on existing data, HIV infection surveillance, performance of standard precaution, and post exposure prophylaxis with anti-retroviral drugs outlined in this review article represent reasonable interim approaches to this complex problem.

\section{Acknowledgements}

I wish to thank professor Manoochehr Makvandi for her kind consultation in the virology section and the late $\mathrm{Dr}$ Mehrdad Borhani for his guidance in clinical epidemiology of HIV/AIDS.

\section{Authors' Contribution}

SM Alavi; design 80\%, writing 90\%, revision $100 \%$, search $70 \%$. MH Sarmast; design $20 \%$, writing $10 \%$, search $30 \%$.

\section{Financial Disclosure}

We have no financial interests related to the material in this manuscript.

\section{Funding/Support}

This study was supported in part by Infectious and Tropical Research Center of Jundishapur University of Medical Sciences.

\section{References}

1. Rahimi-Movaghar A, Amin-Esmaeili M, Haghdoost AA, Sadeghirad B, Mohraz M. HIV prevalence amongst injecting drug users in Iran: a systematic review of studies conducted during the decade 1998-2007. Int J Drug Policy. 2012;23(4):271-8.

2. Rahimi-Movaghar A, Razaghi EM, Sahimi-Izadian E, Amin-Esmaeili M. HIV, hepatitis C virus, and hepatitis B virus co-infections among injecting drug users in Tehran, Iran. Int J Infect Dis. 2010;14(1):e28-33.

3. Alavi SM, Etemadi A. HIV/HBV, HIV/HCV and HIV/HTLV-1 co infection among injecting drug user patients hospitalized at the infectious disease ward of a training hospital in Iran. PakJ Med Sci. 2007;23(4):510.

4. Alavi SM, Alavi L. Seroprevalence study of HCV among hospitalized intravenous drug users in Ahvaz, Iran (2001-2006). J Infect Public Health. 2009;2(1):47-51.

5. Alavi SM, Hajiani E. Hepatitis C infection: a review on epidemiology and management of occupational exposure in health care workers for general physicians working in Iranian health net- work setting. Jundishapur J Microbiol. 2011;4(1):1-9.

6. Hajiani E, Alavi SM. A review on epidemiology, diagnosis and treatment of hepatitis D virus infection. Jundishapur J Microbiol. 2011;4(2):S1-S8.

7. Khosravi AD, Dezfulian A, Alavi SM. Detection of Isoniazid and Rifampin resistant Mycobacterium tuberculosis isolated from tuberculosis patients using conventional method and PCR. Pak JMed Sci. 2006;22(1):47.

8. Alavi SM, Salami N. The causes of death among patients with tuberculosis in Khuzestan, Iran. PakJMed Sci. 2008;24(2):217.

9. Alavi SM, Nadimi M, Shokri S, Zamani GA. Latent tuberculosis infection in individuals with human immunodeficiency virus infection: comparison of tuberculin skin test to the anti TB-IgM antibodies. Pak JMed Sci. 2010;26:11-14.

10. Alavi SM, Albaji A, Sharifi M, Tarrahomi F, Zamani A. Drug induced hepatitis among Iranian patients suffering from tuberculosis. PakJ Med Sci. 2011;27(2).

11. Alavi SM, Khosravi AD, Seyedian SS. Mycobacterium tuberculosis infection: data based review on Tuberculosis in Khuzestan, Iran. Res J Microbiol. 2011;6(10):724-734.

12. Alavi SM, Ahmadi F, Zargari N. [The main risk factors of pulmonary tuberculosis acquisition in hospitalized patients in Razi hospital, Ahvaz-Iran (2001-07)]. J Gorgan Univ Med Sci. 2012;14(2):107-112.

13. Alavi SM, Soltani MH. Study of urethritis among subjects regardless to religious rites. PakJ Med Sci. 2010;26(4):946-949.

14. Alavi SM, Alavi L, Jaafari F. Outbreak investigation of needle sharing-induced malaria, Ahvaz, Iran. Int J Infect Dis. 2010;14(3):e2402.

15. Eshrati B, Asl RT, Dell CA, Afshar P, Millson PM, Kamali M, et al. Preventing HIV transmission among Iranian prisoners: initial support for providing education on the benefits of harm reduction practices. Harm Reduct J. 2008;5:21.

16. Mansoori D, Alaei K, Alaei A. Prevalence of clinical tuberculosis in HIV infected patients from Kermanshah Province, Iran. Tanaffos. 2002;1(2):27-33.

17. Iguchi MY, Bux DA, Kushner H, Lidz V. Correlates of HIV risk among female sex partners of injecting drug users in a highseroprevalence area. Eval Program Plan. 2001;24(2):175-185.

18. Strathdee SA, Philbin MM, Semple SJ, Pu M, Orozovich P, Martinez $\mathrm{G}$, et al. Correlates of injection drug use among female sex workers in two Mexico-U.S. border cities. Drug Alcohol Depend. 2008;92(1-3):132-40.

19. Gyarmathy VA, Neaigus A. The relationship of sexual dyad and personal network characteristics and individual attributes to unprotected sex among young injecting drug users. AIDS Behav. 2009;13(2):196-206.

20. Report on the global AIDS epidemic. 2010. Available from: http:|| www.unaids.org/globalreport/documents/20101123_GlobalReport_full_en.pdf.

21. Alavi SM. Relative frequency of infections among hospitalized injecting drug user-HIV positive patients in Razi hospital, Ahvaz, SW Iran (2001-2003). Jundishapur J Microbiol. 2008;2008(1, Winter):6-9.

22. Mokri A. Brief overview of the status of drug abuse in Iran. Arch Iranian Med. 2002;5(3):184-90.

23. Razzaghi EM, Rahimi MA, Hosseini M, Madanei S. A Rapid Situation Assessment of Drug Abuse in Iran. Tehran: UNDCP \& Welfare Organization; 2001. Available from: http://www.unodc.org/pdf/ iran/publications/RSA 2000 SUMMARY.pdf.

24. Lean. 2011. Available from: http://en.Wikipedia.Org/wiki/Lean.

25. Asadi-Lari M, Sayyari AA, Akbari ME, Gray D. Public health improvement in Iran--lessons from the last 20 years. Public Health. 2004;118(6):395-402.

26. Diot P, Careal M. Global Prespection of Human Immunodeficiency Virus infection and Acquired Immunodeficiency Syndrome. In: Mandell GL, Bennett JE, Dolin R s. Principles and Practice of Infectious Diseases. 7 ed. Philadelphia: Churchill Livingston; 2010. p.1619-1639.

27. AIDS epidemic update.Geneva: UNAIDS/WHO; 2003.

28. Ministry of Health and Medical Education [AIDS/HIV Surveil- 
lance Report]. 2005.

29. Mathers BM, Degenhardt L, Phillips B, Wiessing L, Hickman M, Strathdee SA, et al. Global epidemiology of injecting drug use and HIV among people who inject drugs: a systematic review. Lancet. 2008;372(9651):1733-45.

30. Abu-Raddad L, Akala FA, Semini I, Riedner G, Wilson D, Tawil $\mathrm{O}$, et al. Characterizing the HIV/AIDS epidemic in the Middle East and North Africa: Time for Strategic Action.Middle East and North Africa HIV/AIDS Epidemiology Synthesis Project: World Bank, UNAIDS/WHO; 2010

31. Pisani E, Lazzari S, Walker N, Schwartlander B. HIV surveillance: a global perspective. J Acquir Immune Defic Syndr. 2003;32 Suppl 1:S3-11.

32. Jahani MR, Kheirandish P, Hosseini M, Shirzad H, Seyedalinaghi SA, Karami N, et al. HIV seroconversion among injection drug users in detention, Tehran, Iran. AIDS. 2009;23(4):538-40.

33. Epidemiological software and tools. 2008. Available from: http:// www.unaids.org/en/KnowledgeCentre/HIVData/Epidemiology/epi_software2007.asp.

34. Mercer CH, Hart GJ, Johnson AM, Cassell JA. Behaviourally bisexual men as a bridge population for HIV and sexually transmitted infections? Evidence from a national probability survey. Int J STD AIDS. 2009;20(2):87-94.

35. [AIDS/HIV Surveillance Report]. 2012.

36. Blanchard JF, Khan A, Bokhari A. Variations in the population size, distribution and client volume among female sex workers in seven cities of Pakistan. Sex Transm Infect. 2008;84 Suppl 2:ii24-7.

37. Obermeyer CM. HIV in the Middle East. BMJ. 2006;333(7573):8514.

38. Khawaja ZA, Gibney L, Ahmed AJ, Vermund SH. HIV/AIDS and its risk factors in Pakistan. AIDS. 1997;11(7):843-8.

39. Strategies to support the HIV related needs of refugees and host populations.Geneva: UNAIDS/UNHRC; 2005.

40. MacDonald MA, Elford J, Kaldor JM. Reporting of occupational exposures to blood-borne pathogens in Australian teaching hospitals. Med J Aust. 1995;163(3):121-3.

41. Popovici F, Apetrei RC, Zolotusca L, Beldescu N, Calomfirescu A, Jezek Z, et al. Acquired immunodeficiency syndrome in Romania. Lancet. 1991;338(8768):645-649.

42. Gesielon C, Marcanos D, Ou C. Transmission of HIV in a dental practice. Ann Intern Med.1992;116:798-805.

43. Sterling TR, Chaison RE. General Clinical Manifestation of Human Immunodeficiency Virus infection. In: Mandell GL Bennett JE, Dolin R. Principles and Practice of Infectious Diseases. 7 ed. Philadelphia: Churchill Livingston; 2010.

44. Heris A, Maher D, Graham S, Paulnar M, Gilks C, Ghazi S, et al. [TB and AIDS. Ministry of Health and Medical education].; 2006.

45. Dewar R, Goldstein D, Maldarelli F. Diagnosis of Human Immunodeficiency Virus infection. In: Mandell GL, Bennett JE, Dolin R s. Principles and Practice of Infections Diseases. 7 ed. Philadelphia: Churchill Livingston; 2010. p. 1663-1686.

46. Stewart G Ministry of Health and Medical education [Care of HIV patients]. 2001. 